PET images of pretargeted antibodies showed co-localisation of LO9 and LO1 at bifurcations of the aorta. The first studies of in vivo pretargeted PET detection of atherosclerosis were performed, our studies successfully demonstrated pretargeting of native low-density lipoprotein within atherosclerotic plaques in mice, in-vivo and ex-vivo using plaque specific antibodies. Conflict of Interest none

\section{THE EFFECT OF ENDURANCE EXERCISE ON RIGHT VENTRICULAR MORPHOLOGY IN FEMALE MASTER ATHLETES}

${ }^{1}$ Uchenna Ozo, ${ }^{2}$ Stathis Papatheodorou, ${ }^{3}$ Charlotte Mahoney, ${ }^{4}$ Zephryn Fanton, ${ }^{4}$ Paulo Bulleros, ${ }^{4}$ Michael Papadakis, ${ }^{4}$ Sanjay Sharma. ${ }^{1}$ Cardiology Clinical Academic Group, St. George's University Hospitals NHS Foundation Trust, London, UK; ' Unit of Inherited and Rare Cardiovascular Diseases, Onassis Cardiac Surgery Centre, Greece; ${ }^{3}$ St Mary's Hospital, Manchester University Hospitals NHS Foundation Trust, Manchester Academic Health; ${ }^{4}$ St George's Hospital, London

\subsection{6/heartjnl-2021-BCS.161}

Objectives To investigate the effect of lifelong exercise on right heart size and function in a large group of female master athletes.
Background The benefits of moderate exercise are well known, however several studies have raised concerns about the association between excessive endurance exercise and adverse right ventricular (RV) cardiac remodelling.During exercise the RV undergoes a dramatic increase in preload leading to a 30 -fold increase in RV wall stress. Critically, the reduction in pulmonary vascular resistance pales in comparison to that of systemic resistance leading to an exponential increase in pulmonary artery pressure. Subsequently, intensive endurance exercise has been associated with transient RV dilatation and systolic dysfunction. Furthermore, animal studies and several small cohorts of seemingly healthy predominately male endurance athletes have suggested that repetitive intensive exercise leads to an increased prevalence of right ventricular dysfunction. Few large studies have specifically assessed right heart morphology and function in lifelong female master athletes ( $>40$ years old).

Methods 173 apparently healthy and asymptomatic female master athletes (mean age $55.1 \pm 7.4$ years) underwent echocardiography using conventional planes as recommended by the British Society of Echocardiography. Athletes exercised $>6$ hours a week for at least 10 consecutive years. The results were compared to 43 sedentary age matched controls who exercised less than 3 hours per week (table 1).

Abstract 164 Table 1 Demographics of female athetes and controls

\begin{tabular}{|l|l|l|l|}
\hline & $\begin{array}{l}\text { Female Athletes } \\
\mathbf{N = 1 7 3}\end{array}$ & $\begin{array}{l}\text { Controls } \\
\mathbf{N}=\mathbf{4 2}\end{array}$ & p value \\
\hline Age (years) & $55.1 \pm 7.44$ & $54 \pm 7.32$ & \\
\hline BMI & $21.6 \pm 2.08$ & $25 \pm 3.89$ & $<0.0001^{*}$ \\
\hline Height (cm) & $166.7 \pm 6.37$ & $164.3 \pm 7.39$ & $0.037^{*}$ \\
\hline Weight (kg) & $60.3 \pm 7.5$ & $68.6 \pm 13.6$ & $<0.0001^{*}$ \\
\hline Framingham score (\%) & $2.3 \pm 2.0$ & $3.0 \pm 1.8$ & $0.0065^{*}$ \\
\hline
\end{tabular}

Abstract 164 Table 2 Right heart dimensions and function assessed by 2D echocardiography in female master athletes compared to healthy sedentary controls

\begin{tabular}{|l|l|l|l|}
\hline & $\begin{array}{l}\text { Female Athletes } \\
\mathbf{N}=\mathbf{1 7 3}\end{array}$ & $\begin{array}{l}\text { Controls } \\
\mathbf{N}=\mathbf{4 2}\end{array}$ & p value \\
\hline Age (years) & $55.1 \pm 7.44$ & $54.0 \pm 7.32$ & \\
\hline BMI & $21.6 \pm 2.08$ & $25.0 \pm 3.89$ & $<0.0001^{*}$ \\
\hline RVOT proximal (mm) & $29.8 \pm 4.4$ & $29.4 \pm 3.3$ & 0.6 \\
\hline RVOT distal (mm) & $21.6 \pm 3.9$ & $21.3 \pm 3.5$ & 0.7 \\
\hline RVD 1 (mm) & $38.4 \pm 4.0$ & $35.9 \pm 3.8$ & $0.0005^{*}$ \\
\hline RVD 2 (mm) & $26.8 \pm 3.8$ & $25.5 \pm 3.5$ & 0.057 \\
\hline RVD 3 (mm) & $78.6 \pm 9.0$ & $74.0 \pm 8.2$ & $0.003^{*}$ \\
\hline $\begin{array}{l}\text { RV Area diastole } \\
\text { indexed (mm/m2) }\end{array}$ & $10.9 \pm 2.4$ & $9.0 \pm 1.6$ & $<0.0001^{*}$ \\
\hline RVFAC \% & $48.0 \pm 9.5$ & $49.7 \pm 7.9$ & 0.186 \\
\hline TAPSE & $24.7 \pm 4.5$ & $22.3 \pm 3.1$ & $0.0032^{*}$ \\
\hline $\begin{array}{l}\text { Right atrial area } \\
\text { indexed (mm/m2) }\end{array}$ & $9.9 \pm 1.7$ & $7.6 \pm 1.4$ & $<0.0001^{*}$ \\
\hline $\begin{array}{l}\text { BMI; Body Mass Index, RVD; right ventricular dimension, RVFAC; right ventricular fractional area } \\
\text { change, RVOT; right ventricular outflow tract, TAPSE; tricuspid annular plane systolic excursion. } \\
\text { *Statistically significant }\end{array}$ & & \\
\hline
\end{tabular}


Results Female master athletes demonstrated significantly increased absolute and indexed right ventricular dimensions compared to sedentary controls, with the greatest increases in basal and longitudinal measurements. Athletes also demonstrated a significantly larger right atrial area. Athletes had increased longitudinal RV systolic function based on TAPSE. There was no significant difference in RV fractional area change between athletes and controls. None of the study group revealed regional wall motional abnormalities or dyskinetic RV segments (table 2).

Conclusions Female master athletes continue to demonstrate echocardiographic features of cardiac adaptation into their later years. Chronic endurance exercise is associated with increased right heart size, but no obvious evidence features of arrhythmogenic right ventricular cardiomyopathy. Larger studies including cardiac magnetic resonance scanning for fibrosis and cardiac monitoring are required to identify potential subclinical features of adverse RV remodelling.

Conflict of Interest None

\section{LOGISTICAL DEMAND OF RUNNING A HIGH-VOLUME MRI SERVICE FOR PATIENTS WITH CARDIAC IMPLANTABLE ELECTRONIC DEVICES: FINDINGS FROM A 'ONE-STOP' SERVICE MODEL}

Benjamin Dowsing, Anish Bhuva, Lizette Cash, Emily Webb, James Moon, Charlotte Manisty. Barts Health NHS Trust, London, UK

\subsection{6/heartjnl-2021-BCS.162}

Introduction Patients with cardiac implantable electronic devices (CIEDs) should have access to Magnetic Resonance Imaging (MRI) when needed. Patients are still less likely to be referred and hospitals may not provide a service. A major barrier is reducing the logistical demand required at scale for a safe service. We aimed to quantify the logistical requirements of a high-volume Cardiac Device-MRI service.

Methods A single centre retrospective audit of a high-volume Cardiac Device-MRI service in a tertiary cardiac imaging unit in the UK. Six months of consecutive referrals from September 2020 were reviewed for patient and CIED details and barriers met. Referrals were sorted by source, indication, MRConditional labelling and referrer specialty.

Results 116 MRIs (48\% cardiac, 52\% non-cardiac) were performed on CIED patients in six months (table 1). 53\% were external referrals, $11 \%$ inpatient and $25 \%$ were suspected malignancy. Referrers were $47 \%$ cardiologists and 53\% other specialty. Time from referral to scan was 15 days (interquartile range, IQR: 8 - 32). There were no complications.70\% of referrals contained complete CIED details and 34\% identified the CIED MR labelling. 17\% were referred with incorrect MR-Conditional labelling and $8 \%$ with incorrect non-MR Conditional labelling. 7 additional days were required to obtain complete CIED details, 10\% had delays over 2 weeks (0-145 days). A cardiac physiology department was contacted for $54 \%$, involving 2 departments in $27 \%$. For cancer referrals, obtaining correct details took 1 day longer compared to other referrals and required 2 extra emails to maintain provision within the national time to treatment targets of 62 days. Missing data was similarly present in referrals from Cardiologists and non-Cardiologists (59\% versus 61\% respectively). The non-Cardiologists recorded more incorrect CIED details (8\% vs $0 \%$ ) (figure 1 ).

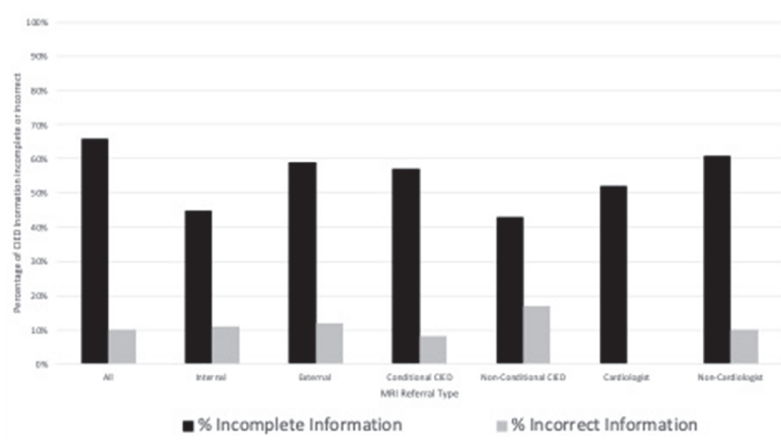

Abstract 165 Figure 1

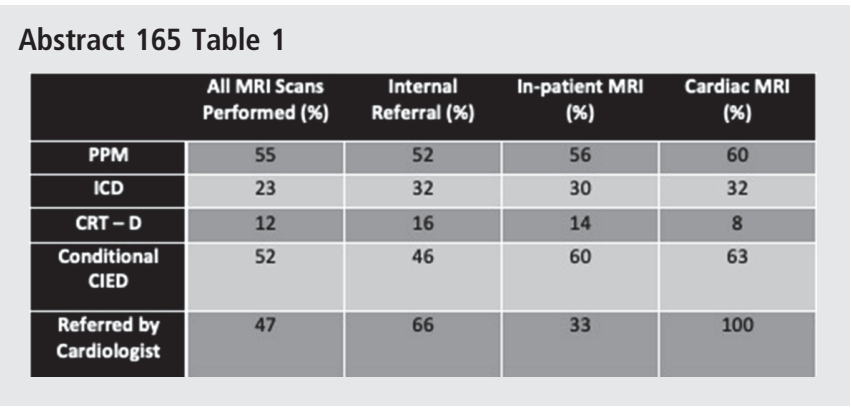

External referrals required 17 days $(11-42)$, compared to 14 (6 -35) days for internal referrals to obtain CIED information. Missing data was similarly present in external and internal referrals (67\% versus $64 \%)$, and $35 \%$ required 3 or more repeat discussions with referrers after initial referral. Patients with non-MR Conditional CIED took 14 days longer to obtain complete referral details than MR-Conditional CIEDs. Even when referrers were aware of non-MR Conditional labelling, 41\% required further discussion between patient and referrer regarding risks and benefits of MRI scanning.

Conclusions Both cardiology and non-cardiology referrers of patients with cardiac implantable electronic devices to MRI incorrectly classify MR-Conditional labelling. There is a large logistical burden to maintaining an MRI service for patients with CIEDs and may explain why some patients are not referred for MRI when required. An online referrals platform is under development to streamline this process, and institutional registration is available at www.mrimypacemaker.com. Conflict of Interest Nil

\section{CT CORONARY ANGIOGRAPHY VERSUS CT FRACTIONAL FLOW RESERVE: A SINGLE CENTRE EXPERIENCE}

Mohaned Egred, Hannah Sinclair, Reuben Loi Yongli, Mohamed Farag, Anna Beattie. Freeman Hospital, Newcastle upon Tyne, UK

\subsection{6/heartjnl-2021-BCS.163}

Background Computerised tomography coronary angiography and fractional flow reserve (CTCA and CT-FFR) are non-invasive diagnostic tools for the detection of flow limiting coronary artery stenoses. Although their negative predictive values are well established, there is a concern that the high sensitivity of these tests may lead to overestimation of coronary artery disease (CAD) and unnecessary invasive coronary angiography (ICA). We compared the positive predictive value (PPV) of 